ISSN 2328-353X

\title{
Diabetes and glycemia as predictors of peri-operative risk: a paradoxically under-characterized relationship
}

Editorial

Nohra EA, Raad M*, Kanj AN, Zeidan A, Kobeissy F

Faculty of Medicine, American University of Beirut Medical Center, Beirut, Lebanon

\section{*Corresponding Author:}

Mohamad Raad,

Faculty of Medicine, American University of Beirut Medical Center, Beirut, Lebanon.

Tel: +961.1 .350000 , ext: 4806

Mobile: +961.70 .165156$

E-mail: mar18@aub.edu.lb

Received: February 15, 2014

Published: March 3, 2014

Citation: Raad M et al (2014) Diabetes and Glycemia as Predictors of Peri-Operative Risk: A Paradoxically Under-Characterized Relationship. Int J Diabetol Vasc Dis Res. 2(2e), 1-2. doi: http://dx.doi. org/10.19070/2328-353X-140007e

Copyright: Raad $\mathbf{M}^{\odot} 2014$ This is an open-access article distributed under the terms of the Creative Commons Attribution License, which permits unrestricted use, distribution and reproduction in any medium, provided the original author and source are credited.

Diabetes mellitus has been recognized as a risk factor for cardiovascular disease since the Framingham study in 1985[1]. Diabetes predisposes to silent myocardial infarction and is traditionally associated with increased cardiovascular risk, including peri-operative cardiac risk. This association is reflected in the Goldman index put forth in 1977 that stratifies diabetes as an intermediate risk factor for poor outcome [2] and is still accepted today as a predictor of cardiac death and morbidity after operations [3].

The relationship between diabetes and adverse postsurgical outcome is well known. A new relationship between hyperglycemia and adverse outcome has come forth and highlights poorer outcomes for those presenting with acute illness and high glucose levels. This relationship is interestingly stronger in non-diabetics. For instance, patients presenting with acute myocardial infarction in addition to hyperglycemia have worse outcomes than those who do not, and this effect is greater non-diabetics [4]. The correction of hyperglycemia with insulin in patients with coronary artery disease may not reduce mortality as evidenced by the DIGAMI trial [5]. Since critically ill patients with hyperglycemia on admission are more likely to die [6], we suggest that it is may be a marker of increased severity of illness, as was thought before the Van den Berghe trial [7]. At this point we cannot conclude that controlling glucose will not remedy this increased risk associated with hyperglycemia, but studies are pointing to an effect beyond what is correctable with insulin. A recent very large trial shows a $\mathrm{U}$-shaped relationship between glycemia and outcome in patients with coronary artery disease [8]. The U-shape indicates that those with both high and low glucose levels fare worse, but disappointingly, the moderate correction of the fasting glucose higher or lower did not modify outcome [8].

Glucose and outcome have a similar relationship in intensive care medicine, as it is another situation where hyperglycemia itself, and it seems especially in the absence of diabetes, is a harbinger of poor outcome [9]. Hypoglycemia was also associated with poor outcome as well [10]. This is indeed similar to the U-shape in cardiac disease, and a similar relationship exists in patients with traumatic brain injury [11]. Consequently, in many situations of severe stress, glycemia at both ends of the glucose scale was associated with poorer outcome.

There must be an explanation for why both high and low glucose levels are associated with poorer outcome other than simply control of diabetes because non-diabetic persons with disturbance of glycemia fare worse[4,9]. Perhaps the best explanation lies in understanding the effect of glucose levels on inflammation. It is well known that hypoglycemia can have an adverse effect on cardiovascular risk because it can precipitate inflammation, endothelial dysfunction, coagulation abnormalities and dysthythmias[12]. Hyperglycemia may also have an adverse effect on inflammation under the effect of stress hormones [13] and the relative deficiency of the anti-inflammatory properties of insulin [14]. We suppose there may be a relationship between glucose and healing in severe stress after surgery which is not directly related to glucose levels but to relative insulin deficiency [13], in which insulin plays a pivotal role in maintaining a balanced inflammatory response. If a person post-surgery does not mount an appropriate inflammatory response, sepsis may set in and there is an exacerbation of morbidity and mortality from sepsis and wound complications in patients with hyperglycemia. On another hand, if that response does not recede in a timely fashion, late death may occur due to the complications of SIRS and sepsis. Lessons learned from trauma indicate that intensive care medicine outcome is strictly dependent on the initial immune response and on its subsequent dampening. Here, the anti-inflammatory properties of insulin may be important [15]. Thus, there are many instances where glucose and relative insulin level may exert an altering effect on the inflammatory process.

We know that diabetes is associated with elevated glucose levels in addition co-morbidities that by themselves worsen outcome such as hypertension, kidney disease, and pulmonary disease. It is believed that the risk of diabetes itself is compounded by the cumulative risk of the associated co-morbidities. Inflammation would theoretically also worsen the effect of those co-morbidities, and this is especially true in patients with poorer lung function due to diabetes-related vasculopathy [16]. Weaning in intensive care would be harder, and the effect inflammatory process in the lungs would be greater[16].

Current protocols pay special attention to the management of peri-operative diabetes medications including oral medications and insulin. However, only a few follow specific protocols for those without overt diabetes. In fact, disorders in glucose metabo- 
lism may be unapparent in situations of normal stress and may become apparent when stress levels increase during acute illness. In context, it may be interesting to study outcomes in a subgroup of patients with subclinical diabetes. Furthermore, a study of glucose tolerance identifies another special subgroup of patients with poor handling of glucose, but these may have normal fasting levels and may or may not behave like diabetics when the stress level is high but they are nil per os. Therefore, both the baseline glucose tolerance and the effect of stress on glucose levels in a particular individual may be parameters worth studying in a perioperative patient.

A traditional way to study diabetes is through animal models. Those models are however intrinsically limited given that they may capture only specific aspects of diabetes and because we now know that diabetes in humans may be a slightly. Further models are needed to model different aspects of the disease including inflammation. The current models certainly are useful to model hyperglycemia [17]. The results of such studies should, however, be interpreted with caution because the process of inflammation in diabetics and the effect of diabetes on the vascular system seems to be different in humans. Perhaps, more knowledge can be extrapolated by studying specifically inflammation in situations of stress as well.

Perhaps the relationship between glycemia-insulin and outcome peri-operatively goes further to encompass the effects on the immune system. We need to study outcomes in both diabetic patients and non-diabetic patients because of the theorized differences in inflammatory responses. In addition to tailoring perioperative glucose-insulin management protocols to the severity of diabetes itself, we feel that after studying the inflammatory process in relation to insulin and glycemia, the results should factor into our management of peri-operative glycemia. New studies are now concentrating on detecting the balance between insulin availability and glucose metabolism [18]. Perhaps the interchange can further be studied in relation to its effect on the level of inflammatory up or down-regulation. Since we know that glucose affects inflammation, we should also study that relationship itself in post-surgical stress to best understand it and its effect on outcome and to develop protocols to control the outcomes.

\section{References}

[1]. Kannel WB. Lipids, diabetes, and coronary heart disease: insights from the Framingham Study. American heart journal. 1985;110:1100-1107.

[2]. Goldman L, Caldera DL, Nussbaum SR, et al. Multifactorial index of cardiac risk in noncardiac surgical procedures. The New England journal of medicine. 1977;297:845-850.

[3]. Tao LS, Mackenzie CR, Charlson ME. Predictors of postoperative complications in the patient with diabetes mellitus. Journal of diabetes and its complications. 2008;22:24-28.

[4]. Ainla T, Baburin A, Teesalu R, Rahu M. The association between hyperglycaemia on admission and 180-day mortality in acute myocardial infarction patients with and without diabetes. Diabetic medicine : a journal of the British Diabetic Association. 2005;22:1321-1325.
[5]. Malmberg K, Ryden L, Efendic S, et al. Randomized trial of insulin-glucose infusion followed by subcutaneous insulin treatment in diabetic patients with acute myocardial infarction (DIGAMI study): effects on mortality at 1 year. Journal of the American College of Cardiology. 1995;26:57-65.

[6]. Umpierrez GE, Isaacs SD, Bazargan N, You X, Thaler LM, Kitabchi AE. Hyperglycemia: an independent marker of in-hospital mortality in patients with undiagnosed diabetes. The Journal of clinical endocrinology and metabolism. 2002;87:978-982.

[7]. van den Berghe G, Wouters P, Weekers F, et al. Intensive insulin therapy in critically ill patients. The New England journal of medicine. 2001;345:13591367.

[8]. Yang SW, Zhou YJ, Tian XF, et al. Association of dysglycemia and all-cause mortality across the spectrum of coronary artery disease. Mayo Clinic proceedings. 2013;88:930-941.

[9]. Rady MY, Johnson DJ, Patel BM, Larson JS, Helmers RA. Influence of individual characteristics on outcome of glycemic control in intensive care unit patients with or without diabetes mellitus. Mayo Clinic proceedings. 2005;80:1558-1567.

[10]. Investigators N-SS, Finfer S, Chittock DR, et al. Intensive versus conventional glucose control in critically ill patients. The New England journal of medicine. 2009;360:1283-1297.

[11]. Mowery NT, Gunter OL, Guillamondegui O, et al. Stress insulin resistance is a marker for mortality in traumatic brain injury. The Journal of trauma. 2009;66:145-151; discussion 151-143.

[12]. Hirsch IB. Improvements in our understanding of diabetes mellitus. Mayo Clinic proceedings. 2013;88:907-909.

[13]. Robinson LE, van Soeren MH. Insulin resistance and hyperglycemia in critical illness: role of insulin in glycemic control. AACN clinical issues. 2004;15:45-62.

[14]. Dandona P, Chaudhuri A, Mohanty P, Ghanim H. Anti-inflammatory effects of insulin. Current opinion in clinical nutrition and metabolic care. 2007; 10:511-517.

[15]. Olefsky JM, Glass CK. Macrophages, inflammation, and insulin resistance. Annual review of physiology. 2010;72:219-246.

[16]. Kaparianos A, Argyropoulou E, Sampsonas F, Karkoulias K, Tsiamita M, Spiropoulos K. Pulmonary complications in diabetes mellitus. Chronic respiratory disease. 2008;5:101-108.

[17]. P. J. Little NO, N. Cohen. Understanding Diabetes and Its Complications for Biomedical Research. International Journal of Diabetology \& Vascular Disease Research 2013.

[18]. Pretty CG, Le Compte AJ, Chase JG, et al. Variability of insulin sensitivity during the first 4 days of critical illness: implications for tight glycemic control. Annals of intensive care. 2012;2:17. 